\title{
Representaciones sociales sobre educación religiosa escolar en una sociedad plural*
}

Fredy Alonso Quintero Torres

https://orcid.org/0000-0002-7922-4896

Universidad Santo Tomás, Colombia

fredyquintero@ustadistancia.edu.co

\section{Resumen}

Este artículo presenta la ruta metodológica, los hallazgos y los resultados de un trabajo investigativo sobre las representaciones sociales (RS) de la clase de educación religiosa escolar (ERE). Lo anterior con el fin de vislumbrar y entender los componentes que estructuran el conocimiento social que se encuentra en las RS que tienen los estudiantes de dos instituciones escolares confesionales de Bogotá sobre la clase de ERE y reconocer los aportes de esta RS en la consolidación de una educación religiosa con enfoque pluralista. El proceso metodológico es mixto y parte de los enfoques de estudio de las RS, asumiendo de modo complementario las perspectivas procesual y estructural, en las que se armonizan técnicas narrativas y asociativas para develar los componentes esenciales de las RS. Los resultados mostraron que las RS sobre la clase de ERE se estructuran principalmente a partir de las nociones de catolicismo y pluralismo y, además, que la perspectiva metodológica mixta relaciona complementariamente los enfoques para la investigación en RS, haciendo posible el conocimiento preciso y consistente de los elementos estructurales de las RS sobre la clase de ERE.

\section{Palabras clave (Fuente: tesauro de la Unesco)}

Educación religiosa; estudios de religión; pluralidad religiosa; religión católica; representaciones sociales.

\footnotetext{
* Este artículo es resultado de la investigación "Representaciones sociales sobre la clase de educación religiosa escolar de los estudiantes de grado 11 de dos instituciones católicas de Bogotá y sus implicaciones en la construcción de una ERE pluralista", desarrollada como trabajo final de la Maestría en Educación de la Universidad Santo Tomás, Bogotá, Colombia.
} 


\title{
Social Representations of Religious Instruction in a Plural Society*
}

\begin{abstract}
This article presents the methodological procedure, findings, and results of a study on the social representations (SR) of religious instruction (RI) classes. It aims to glimpse and understand the components of the social knowledge found in the SRs of RI of students from two confessional schools in Bogota and recognize the contributions of these SRs to consolidating a religious education with a pluralistic approach. The methodological process is mixed and builds on the SR study approaches, supplemented by procedural and structural perspectives that harmonize narrative and associative techniques to reveal the essential components of SRs. The results showed that the $S R$ of RI are structured mainly from Catholicism and pluralism and that the mixed methodological perspective complements the approaches for SR research, making it possible to know precisely and consistently the structural elements of the SRs of RI.
\end{abstract}

\section{Keywords (Source: Unesco Thesaurus)}

Religious education; religious studies; religious pluralism; Catholicism; social representations.

This article derives from the research "Representaciones sociales sobre la clase de educación religiosa escolar de los estudiantes de grado 11 de dos instituciones católicas de Bogotá y sus implicaciones en la construcción de una ERE pluralista" conducted as part of the MA in Education thesis, Universidad Santo Tomás, Bogotá, Colombia. 


\section{Representações sociais sobre ensino religioso escolar numa sociedade plural*}

\section{Resumo}

Este artigo apresenta a rota metodológica, os achados e os resultados de um trabalho de pesquisa sobre as representações sociais (RS) da aula de ensino religioso escolar (ERE). O objetivo é vislumbrar e entender os componentes que estruturam o conhecimento social que se encontra nas RS que têm os estudantes de duas instituições escolares confessionais de Bogotá sobre a aula de ERE e reconhecer as contribuições desta RS na consolidação de um ensino religioso com abordagem pluralista. O processo metodológico é misto e parte das abordagens de estudo das RS, assumindo de modo complementar as perspectivas processual e estrutural, nas quais são harmonizadas técnicas narrativas e associativas para evidenciar os componentes essenciais das RS. Os resultados mostraram que as RS sobre a aula de ERE são estruturadas principalmente a partir das noções de catolicismo e pluralismo, além disso que a perspectiva metodológica mista relaciona complementarmente as abordagens para a pesquisa em RS, tornando possivel o conhecimento preciso e consistente dos elementos estruturais das RS sobre a aula de ERE.

\section{Palavras-chave (Fonte: Tesauro da Unesco)}

Ensino religioso; estudos de religião; pluralidade religiosa; religião católica; representações sociais.

* $\quad$ Este artigo é resultado da pesquisa "Representações sociais sobre a aula de ensino religioso dos estudantes do último ano do ensino médio de duas instituições católicas de Bogotá e suas implicações na construção de um ERE pluralista", desenvolvida como trabalho final do mestrado em Educação da Universidad Santo Tomás, Bogotá, Colômbia. 
La clase de educación religiosa escolar (ERE) se ha visto enfrentada a una serie de transformaciones en el entorno colombiano, con la Constitución Política colombiana de 1991, documento que estableció un distanciamiento entre la esfera político-social y la religiosa (Moncada y Pérez, 2020). Esta división influyó directamente en el campo educativo, pues a partir de este hecho la ERE, que estaba sustentada en la iglesia católica, dejó de ocupar un puesto predilecto, sólido y seguro en la educación colombiana y pasó a asumir un espacio común y, en cierto sentido, opcional, pese a la distinción dada por la ley que reconoce a la ERE como un área esencial e ineludible en la educación integral de los colombianos (Ley 115 de 1994, art. 23).

Frente a esta situación, que concierne a la enseñanza de la ERE, se precisa la problematización de sus fundamentos y legitimidad en el contexto escolar, más allá del sustento dado por la iglesia católica. A su vez, esta discusión posibilitará la búsqueda de otras perspectivas que ensanchen la manera en que se entiende la ERE. En esta indagación por nuevas perspectivas y horizontes, la teoría de las representaciones sociales (TRS) se constituye en una herramienta para continuar profundizando en el análisis teórico sobre la ERE, puesto que permite contemplarla como una realidad social, diferente al enfoque dado por la confesionalidad cristiana católica, en la que también se pueden considerar como válidas las comprensiones y las percepciones de sus protagonistas: los estudiantes.

Así entonces, a partir del contexto actual en el que se desarrolla la ERE, en donde se cuestionan sus objetivos y fundamentos y se pasa de la uniformidad y la homogeneidad al pluralismo y la heterogeneidad, especialmente en el ámbito religioso (Coy, 2010), se hace necesario buscar y repensar la ERE atendiendo a otros lugares que consideren e incluyan la riqueza espiritual, religiosa y cultural de la humanidad, así como a los sujetos y los contextos en los que se enseña (Cantillo y Quintero, 2020).
En este sentido, el objetivo que se propone es vislumbrar y comprender las representaciones sociales (RS) que tienen los estudiantes sobre la ERE en contextos confesionales y a su vez reconocer los aportes de estas RS en la construcción de una ERE en perspectiva pluralista. Para dar razón de lo anterior, el texto se estructura en tres grandes partes: primera, una aproximación al estado del arte sobre ERE y TRS, identificando puntos de relación; segunda, la metodología del estudio; y tercera, el análisis y discusión de los resultados, a su vez subdividido en tres momentos: análisis del contexto de las RS, resultados desde el enfoque estructural y procesual para el estudio de las RS y, finalmente, las RS sobre la clase de ERE.

\section{Hacia una relación entre la ERE y la TRS}

El paradigma confesional cristiano católico ha predominado en la compresión de la ERE en Colombia. Así, por ejemplo, los estándares propuestos por la Conferencia Episcopal de Colombia (2017) para la enseñanza de la ERE siguen siendo criterios aceptados y ampliamente usados tanto en instituciones abiertamente confesionales como en las no confesionales. Sin embargo, esta propuesta representa una situación problemática, pues con ella se tiende a asumir que la catequesis, la teología y la educación religiosa definen lo mismo, suponiendo con esto que el contenido de las tres es la doctrina católica. Este modo de comprender la ERE tiende a malinterpretar su esencia, porque, aunque en sus orígenes la ERE estuvo ligada a la evangelización católica, catequesis o iniciación cristiana, su naturaleza no se limita a un mecanismo exclusivo de evangelización cristiana.

En la actualidad se afianza la distinción entre educación religiosa y doctrina católica, de tal manera que se está superando el modelo según el cual la educación religiosa se ha comprendido y cimentado a partir de la religión católica. Esta situación al mismo tiempo hace posible abrir el campo de entendimiento de la educación religiosa hacia una perspectiva aconfesional y pluralista. Trabajos 
investigativos y reflexiones académicas como las de María Elizabeth Coy (2009; 2010), Jaime Bonilla (2013; 2014; 2015), José Luis Meza (2011; 2013), Botero y Hernández (2018), Naranjo y Moncada (2019), Cuéllar y Moncada (2019), Moncada (2020), Cuéllar, Moncada y Valencia (2020) y Pérez et al. (2020) han dado pasos importantes en la reformulación y nueva comprensión de la ERE que la distancie de una enseñanza exclusiva de la doctrina cristiana católica.

Al respecto, resulta de vital importancia continuar reflexionando y repensando la ERE con otros horizontes que consideren, por una parte, la multiplicidad cultural y religiosa junto con los entornos en los que están sumergidos los actores de la ERE, especialmente los estudiantes, y, por otra, los desarrollos investigativos en el campo social y educativo que, a su vez, permiten ampliar la compresión de un fenómeno social y cultural como lo es la religión y, de manera específica, la clase de educación religiosa. Esto con el propósito de justificar y resignificar la enseñanza de la educación religiosa en el contexto escolar y profundizar una dimensión fundamental de la persona que necesita ser desarrollada.

Tanto la religión como la clase de educación religiosa son realidades sociales que implican un conjunto de elementos de orden político, cultural e ideológico que tocan directamente a los actores sociales que se relacionan con ella. Por lo tanto, es valioso y novedoso considerar la ERE en relación con estos factores socioculturales que, al mismo tiempo, permiten comprenderla más allá del paradigma confesional. En esta perspectiva sociocultural se ubica la TRS, la cual se refiere fundamentalmente al conocimiento de sentido común que se construye a partir de la interrelación entre personas, colectivos sociales y componentes de la realidad social, es decir, instituciones, medios de comunicación, legislación, etc. (Moscovici, 1979).

En cuanto conocimiento de sentido común, la TRS expone varios puntos de consideración para los estudios en educación y, de manera especial, para la ERE. En primer lugar, la TRS permite comprender cómo el ser humano construye y a la vez es construido por la realidad social (Banchs, 1988). En este sentido, se comprende que los estudiantes, en cuanto sujetos educativos, no son actores pasivos del proceso de aprendizaje y, según la TRS, ellos son construidos por las relaciones que se dan en el contexto de la clase de ERE, pero no en una relación unidireccional, pues ellos mismos -a través de sus acciones, creencias, prácticas y maneras de comprender la clasela construyen.

En esta perspectiva, Jodelet afirma que en educación "no es suficiente comprender el saber y las consecuencias de su difusión junto con la asimilación en el marco limitado de una relación lineal entre emisión-transmisión-recepción" (2011, p. 138). Esto significa que quien recibe el mensaje no puede ser considerado como un sujeto pasivo en el cual se registra conocimiento e información nueva y, además, que la comunicación no puede seguir caracterizándose por ser una correlación dependiente y jerárquica entre el emisor, poseedor del conocimiento, y el receptor, sujeto paciente y neutral.

Otro punto de relación entre las RS y la educación en la clase de ERE es su carácter contextual. El estudio sobre representaciones sociales se desarrolla en contextos específicos que exponen los conocimientos, las conductas, las prácticas y las experiencias de sujetos que son inscritos e involucrados en roles específicos y en espacios institucionales, sociales y culturales más amplios. Las RS permiten acceder a los diversos aspectos de la realidad del objeto que es apropiado en un momento determinado. Es en este punto donde el enfoque de estudio de las RS se constituye en instrumento y mediación pertinente en el campo educativo, donde "la materia y la vida refieren a prácticas sociales que, en constante evolución en sus objetivos, sus fundamentos, sus valores, son asumidas por sujetos que siempre tendrán la última palabra" (Jodelet, 2011, p. 151).

De lo anterior se deriva un último punto de relación entre la TRS y la ERE: su estrecha relación con las prácticas sociales. Toda RS, para ser tal, debe 
incluir la capacidad de conformar un patrón o contramodelo de conducta que pauta las prácticas sociales (Alasino, 2011). De este modo, la inscripción de los sujetos en un contexto mayor permite que el estudio basado en las RS adquiera gran importancia, en especial para reflexionar sobre la educación y las prácticas formativas (Quintero y Ortiz, 2020), porque estas trascienden la socialización, las prácticas de enseñanza-aprendizaje y el manejo de unas estrategias pedagógicas. En este sentido, "el estudio de los procesos simbólicos que operan al interior de estas prácticas es susceptible de ser abordado y esclarecido por el enfoque de las RS" (Jodelet, 2011, p. 138).

Así pues, un estudio de la ERE basado en la TRS amplía y complejiza el problema de investigación, por varias razones: en primer lugar, porque permite ubicarla en una perspectiva de análisis diferente de la confesional, al tratarse de un estudio que considera elementos sociales. En segundo lugar, como objeto de estudio social, el estudio de la ERE a partir de la TRS hace referencia al conocimiento de sentido común, tipo de conocimiento que desempeña un papel central para comprender la manera en que los sujetos piensan, establecen y se apropian de su vida en determinados contextos sociales. En tercer lugar, la aproximación investigativa basada en las RS permite conocer los procesos de formación del conocimiento social, a través del cual los sujetos construyen y son construidos por la realidad social; finalmente, dado que las RS guían las prácticas o comportamientos de los sujetos en diversos contextos sociales, el conocimiento de su contenido puede generar transformaciones en las prácticas mismas.

\section{Metodología del estudio}

De manera general, la ruta metodológica que se asumió en la investigación consideró los siguientes elementos: se trató de una investigación que se puede denominar "mixta", pues se tomaron de manera complementaria y heurística los enfoques de estudio de las RS - procesual y estructural- (Araya, 2002), mientras que el enfoque epistemológico se sustentó en la hermenéutica, que dialoga coherentemente con los estudios sobre RS, dado que permite comprender a la persona como creadora de sentidos y significados (Pérez, Nieto y Santamaría, 2019). En cuanto al método de investigación, el análisis es plurimetodológico, combinando técnicas asociativas y narrativas para develar las RS, toda vez que hasta ahora no existe una sola técnica que permita recolectar de modo conjunto los componentes de la representación (Abric, 2001).

La ruta metodológica mixta tiene su fundamento en la complementariedad de los enfoques procesual y estructural, con sus respectivas técnicas de corte cualitativo y cuantitativo, reconociendo sus diferencias paradigmáticas, pero destacando sobre todo sus características complementarias. Desde esta perspectiva, se asumieron elementos del enfoque estructural con el propósito de develar la estructura de las RS (elementos del núcleo central y componentes periféricos), y se tomaron elementos de la perspectiva procesual, con la intención de "identificar e interpretar el contenido de las RS como producto de un contexto histórico-social" (Cuevas, 2016, p. 114). Se consideró que esta opción metodológica permite develar y comprender las RS de una manera holística, con el objetivo de justificar rigurosamente los resultados y obtener una perspectiva más amplia del fenómeno estudiado.

Para la recolección de los datos resultaron pertinentes las siguientes técnicas: la asociación libre, la carta asociativa (unificadas en un taller asociativo) y los grupos de discusión. Aunque la asociación libre y la carta asociativa son técnicas de corte cuantitativo originalmente independientes, se asumieron en perspectiva dialógica, dado que ambas, como técnicas asociativas fundadas en la producción verbal, "permiten reducir la dificultad o los límites de la expresión discursiva" (Abric, 2001, p. 59). Con la intención de verificar la centralidad de los elementos obtenidos por medio de las técnicas asociativas, se desarrolló el método del cuestionario de verificación propuesto por Guimelli (2001) y recuperado por Pa- 
rales (2006), como cuestionamiento de centralidad. Con este, los componentes con mayor frecuencia y más rápidamente evocados se cuestionaron en cuanto a su centralidad por medio de expresiones estándar, sobre las que los participantes de la investigación deben escoger las respuestas: Sí, TAL VEZ, NO, acerca de si la afirmación era esencial en referencia a la clase de ERE.

En diálogo con las técnicas asociativas, para la recolección de datos de orden cualitativo se desarrollaron grupos de discusión, en los que se propusieron cinco preguntas que derivaron de las palabras o conceptos que resultaron del taller asociativo y que, posterior a la sistematización y análisis, se propusieron como elementos centrales en la estructura de las RS. La intención de los grupos de discusión consistió en conocer, a través del contenido expresado por cada uno de ellos y plasmado en una guía de trabajo los componentes representativos acerca de la clase de ERE. En este sentido, con las preguntas se buscó conocer qué es, cómo se desarrolla, por qué es importante, qué se enseña en la clase de ERE y qué relación tiene esta con la enseñanza del pluralismo religioso. De manera general, el análisis inicial de los datos expresados en los grupos de discusión se realizó de acuerdo con el contenido temático de los textos finales. Para orientar esta tarea se tuvo como telón de fondo la relación entre las preguntas formuladas a los grupos de discusión y las categorías en torno a las cuales se organizó el conjunto de términos resultantes del taller asociativo, que fueron: lo confesional, lo plural, lo ético-moral y lo académico.

El análisis de la información proporcionada en los grupos de discusión se efectuó de conformidad con algunas de las disposiciones metodológicas propuestas por Taylor y Bogdan (1987, citados por Mireles, 2015): se leyeron las transcripciones de manera cuidadosa y repetida, se hicieron anotaciones al margen y se determinaron temas sobresalientes. También se siguieron las etapas o tareas del análisis de datos cualitativos planteadas por Rodríguez, Lorenzo y Herrera (2005) que son la simplificación de los datos, su disposición y transformación, la obtención de resultados y la verificación de conclusiones.

En concordancia con lo anterior, se retomó uno de los pasos indicados por Gómez (2000) para el análisis cualitativo de contenido, dada su conveniencia para establecer el diálogo entre los enfoques procesual y estructural desarrollados en la investigación. Este paso es el número tres y corresponde a la selección de unidades de análisis. Para la selección de estas unidades se tomaron las tres dimensiones de las RS propuestas por Moscovici: "información, actitud y campo de representación” (1979, p. 45). Para esto, la información se organizó en una matriz mixta que incluyó las tres dimensiones y cada una de las palabras del núcleo central y periféricas. No obstante, se debe aclarar que no se realizó un análisis cualitativo de contenido en sentido estricto, sino que se tomaron algunos elementos que favorecieron el análisis y la compresión de los datos obtenidos mediante las técnicas asociativas y los grupos de discusión.

Otro punto fundamental en el proceso metodológico para la comprensión de las RS sobre la clase de ERE fue el análisis del contexto en que emergen las RS. En correspondencia con lo propuesto por Mireles (2015), la estrategia que se acogió para conocer el contexto fue la revisión documental, a través de la cual se buscaron, leyeron y analizaron numerosos documentos, como textos de carácter legal, políticas públicas y documentos institucionales de los colegios con los que se desarrolló la investigación. Como complemento, y en diálogo con la revisión documental, se aplicó un cuestionario virtual para ampliar la información sobre el contexto familiar y personal de los estudiantes. Este cuestionario se construyó en su gran mayoría a partir de preguntas de selección múltiple, por ejemplo: cuál es su profesión religiosa (protestantismo, catolicismo, islam, judaísmo, etc.).

En cuanto a la selección de los participantes, esta se desarrolló de acuerdo con la disponibilidad de los estudiantes de dos colegios confesionales de la ciudad de Bogotá. La muestra estuvo constituida 
por estudiantes de grado 11, grupo en el cual se contó con la presencia de mujeres y hombres, con un rango de edad entre los 15 y los 18 años, que pertenecen a la clase socioeconómica media y media-alta. Además, se aseguró la presencia de jóvenes que profesaran abiertamente la religión católica, otras religiones no católicas y los que se consideraron no creyentes.

Teniendo en cuenta todo el proceso, y con la intención de facilitar la comprensión del camino metodológico, en términos generales y de manera esquemática, se siguió esta ruta:

- Análisis del contexto en el que emergen y circulan las RS a través de la revisión documental.

- Aplicación del taller de asociación de palabras, desarrollado con las técnicas de la asociación libre y la carta asociativa. Los datos se organizaron y se sistematizaron para explorar e identificar los elementos centrales y periféricos de las RS sobre la clase de ERE (enfoque estructural).

- Después se desarrolló el cuestionario virtual con dos objetivos: ampliar los datos para el análisis del contexto y verificar la centralidad de los elementos constitutivos de las RS.

- A continuación, se desarrollaron los grupos de discusión en torno a preguntas sobre el objeto de la representación y las prácticas cotidianas en clase de ERE (enfoque procesual).

- Finalmente, se leyeron y analizaron cuidadosamente las respuestas. Se triangularon los datos obtenidos y, por su carácter cuantitativo y cualitativo, se tejieron relaciones y se buscaron los sentidos y significados para develar la estructura de las RS sobre la ERE.

\section{Resultados: develamiento de las RS sobre la clase de ERE}

A continuación, se presentan los resultados de la investigación. En primer lugar, se exponen los hallazgos a partir del análisis del contexto social y religioso en el que aparecen y circulan las RS sobre la ERE de los estudiantes de los colegios confesionales que hicieron parte de esta investigación. En segundo lugar, se presentan los resultados del análisis y la interpretación de las RS sobre la clase de ERE, a su vez desarrollada en tres momentos: 1) aproximación cuantitativa a las RS sobre la clase de ERE, 2) aproximación cualitativa y 3) diálogo entre las dos perspectivas para develar finalmente las RS sobre la ERE.

\section{Contexto de aparición de las RS sobre la clase ERE}

Para la comprensión de las RS es esencial construir el contexto en el que se gestan y configuran, porque las RS, como saber de sentido común, mantienen una profunda e íntima relación entre los sujetos que construyen esta forma de conocimiento y el contexto en que aparecen (Mireles, 2015). Al comprender el entorno en el que surge y transita determinada RS sobre la ERE, no se buscó hacer únicamente una descripción del contexto, sino que se procuró analizar un conjunto de acontecimientos y hechos en relación con la clase que justamente permitieran comprender el escenario en el que aparece y circula esa RS sobre la ERE. La estrategia que se adoptó fue la revisión documental (Pérez, Ramírez y Rojas, 2020).

Uno de los primeros documentos que se analizó fue la Constitución Política de Colombia de 1991. Con respecto a la formación de la RS sobre la clase, se encontró que la Constitución Política configura tres características que influyen en la manera de comprender lo que se refiere al campo religioso en el contexto colombiano y, por lo tanto, a la educación religiosa: el pluralismo, la libertad y la igualdad religiosa. El cuerpo estudiantil que participó en la investigación vincula la clase de ERE a un espacio de formación en el que se conocen las diversas religiones presentes en el país y en el mundo, una clase en la que se puede expresar libremente la profesión de fe y en la que todas las religiones deben ser reconocidas por igual, sin privilegios de ningún tipo. 
Otro de los textos que se retomó fue la Ley General de Educación de 1994. En relación con la construcción de la RS se halló que la Ley 115 configura tres características que influyen en la manera de comprender la clase de ERE: su carácter obligatorio, fundamentaly no confesional. Al reflexionar sobre la clase de religión, los estudiantes comprenden que su enseñanza corresponde con una clase que es importante recibir y además es necesaria para alcanzar los objetivos de la educación en el país. Además, en estrecha relación con dicha necesidad e importancia, se reconoce que la clase de ERE no debe ser irreparablemente una clase confesional, donde se enseñe con exclusividad una sola religión.

En relación con el reconocimiento del contexto familiar y personal de los sujetos, se retomaron cinco preguntas formuladas a los estudiantes a través del cuestionario virtual, pues estas permitieron develar elementos significativos en relación con la construcción de las RS. De acuerdo con los resultados del cuestionario, se encontró que: en primer lugar, la mayoría de los sujetos $(88,1 \%)$ provienen y fueron educados en familias en las que se profesa el catolicismo. En segundo lugar, la gran mayoría de los estudiantes son jóvenes creyentes, es decir, que manifestaron creer en Dios (92,9\%). Con respecto al cuestionamiento sobre la importancia de la religión, más de la mitad de los estudiantes dieron una respuesta positiva (59,5\%); en relación con la pregunta sobre la religión que profesaban, la mayoría de los estudiantes afirmaron ser católicos (66,7\%); y finalmente, frente a la pregunta por la identificación y la práctica de la religión que afirmaron profesar, el porcentaje de estudiantes muy identificados, medianamente identificados y practicantes correspondió al 59,5\%.

Los textos que se analizaron para comprender el contexto de aparición de las RS de los estudiantes sobre la clase de ERE, aunque no se incluyeron todos en este documento, por cuestiones prácticas, presentan elementos suficientes para comprender que el contexto de aparición de las RS sobre la clase es el contexto orientado en gran medida por las políticas públicas y legales, por los lineamientos institucionales y por el contexto familiar, todos ellos determinados por una fuerte tensión entre lo confesional y lo plural.

\section{Aproximación al enfoque estructural de las RS sobre la ERE (análisis cuantitativo)}

A continuación, se presentan los hallazgos y los resultados del enfoque estructural para develar los elementos centrales y periféricos de las RS sobre la clase de educación religiosa. A través del taller asociativo se obtuvieron 653 palabras. Sobre esta lista de palabras evocadas por los estudiantes y asociadas a la clase de ERE, se efectuó un primer análisis de frecuencia con el objetivo de determinar el núcleo central y los elementos periféricos de la RS. Si bien la relación entre frecuencia, rango e importancia es la que permite establecer la centralidad de los elementos integrantes de una RS', considerar inicialmente solo la frecuencia permitió abrir el camino para develar las RS.

En la Tabla 1 se presentan los elementos nombrados con mayor frecuencia en los dos colegios (se consideraron las palabras con una frecuencia mínima de 3). A partir de las técnicas asociativas, se encontró que la palabra común a las dos instituciones y con mayor frecuencia (34 veces nombrada) correspondió a Dios; de igual modo, se detectó que las palabras menos frecuentes fueron enseñanza $y$

$1 \quad$ Con base en lo afirmado por Abric (2001), "la congruencia entre estos elementos (frecuencia, rango e importancia) permiten determinar los elementos centrales y periféricos en la estructura de la RS" (p. 6o). En este sentido, determinar estos tres elementos en el grupo de palabras, es fundamental para establecer los elementos centrales de la RS, según el supuesto de que "las palabras más frecuentes, importantes y primeras en la evocación tienen mayor probabilidad de pertenecer al núcleo central" (Sá, 1996, citado por Parales, 2006, p. 616). Según lo anterior, es necesario especificar que la frecuencia hace referencia al número de veces que es usada una palabra en la población, el rango se refiere al lugar de evocación de las palabras y se obtiene sobre el promedio del total de la población que utilizó la palabra y la importancia se refiere al peso semántico que dan las personas a las palabras. 
conocimiento (nombradas 9 y 8 veces, respectivamente). En el costado derecho de la Tabla 1 se resalta el total de las palabras más frecuentes y comunes a los dos colegios. Estas palabras se revisaron para determinar su pertenencia al núcleo central o a los elementos periféricos de las RS sobre la clase de ERE:

Tabla 1. Frecuencia de la palabra en la población

\begin{tabular}{|c|c|c|c|c|c|}
\hline \multicolumn{2}{|c|}{ Colegio 1} & \multicolumn{2}{|c|}{ Colegio 2} & \multicolumn{2}{|l|}{ Total } \\
\hline Dios & 19 & Biblia & 19 & Dios & 34 \\
\hline $\mathrm{Fe}$ & 16 & Dios & 15 & Biblia & 33 \\
\hline Biblia & 14 & Jesús & 10 & Religiones & 23 \\
\hline Religiones & 13 & Vida & 11 & $\mathrm{Fe}$ & 20 \\
\hline Creencias & 12 & Religiones & 10 & Moral, ética y valores & 20 \\
\hline Moral, ética y valores & 11 & Moral, ética y valores & 9 & Creencias & 18 \\
\hline Historia & 9 & Reflexión & 7 & Jesús & 18 \\
\hline Filosofía & 8 & Creencias & 6 & Vida & 18 \\
\hline Jesús & 8 & María & 6 & Historia & 13 \\
\hline Vida & 7 & Puntos de vista & 5 & Reflexión & 10 \\
\hline Formación & 6 & Enseñanza & 4 & Catolicismo & 10 \\
\hline Enseñanza & 5 & Catolicismo & 5 & Enseñanza & 9 \\
\hline Catolicismo & 5 & $\mathrm{Fe}$ & 4 & Conocimiento & 8 \\
\hline
\end{tabular}

Fuente: elaboración propia con base en las respuestas de los estudiantes.

Al combinar la frecuencia, el rango medio de evocación y la importancia de las palabras presentadas, se obtuvo una primera lista con posibles elementos centrales y periféricos. En la Tabla 2 se presentan los posibles elementos centrales y periféricos comunes a los dos grupos, si bien solo se incluyeron los de mayor frecuencia. El análisis reveló unos ele- mentos con frecuencia, rango promedio de evocación y nivel de importancia significativamente altos, que inicialmente fueron considerados centrales en las RS. Asimismo, se descubrieron otros elementos frecuentes, pero con bajos niveles de importancia y/o de evocación tardía, por lo que se consideraron elementos periféricos. 
Tabla 2. Identificación preliminar de elementos centrales y periféricos.

\begin{tabular}{|c|c|c|c|c|}
\hline & Palabra & Frecuencia & Rango & Importancia \\
\hline \multirow{5}{*}{ NúCleO CENTRAL } & Dios & 34 & 4,27 & 29 \\
\hline & Biblia & 33 & 5,52 & 16 \\
\hline & $\mathrm{Fe}$ & 20 & 6,90 & 11 \\
\hline & Creencias & 18 & 6,94 & 14 \\
\hline & Jesús & 18 & 7,61 & 10 \\
\hline \multirow{8}{*}{ ELEMENTOS PERIFÉRICOS } & Religiones & 23 & 6,19 & 7 \\
\hline & Moral, ética y valores & 20 & 8,25 & 6 \\
\hline & Vida & 18 & 6,89 & 4 \\
\hline & Historia & 13 & 8,00 & 6 \\
\hline & Reflexión & 10 & 7,80 & 3 \\
\hline & Catolicismo & 10 & 6,80 & $\mathrm{O}$ \\
\hline & Enseñanza & 9 & 4,78 & 8 \\
\hline & Conocimiento & 8 & 5,50 & 3 \\
\hline
\end{tabular}

Fuente: elaboración propia con base en las respuestas de los estudiantes.

Al revisar en paralelo los indicadores de frecuencia, rango e importancia, se obtienen los elementos que estructuran las RS. Lo que se debe determinar a continuación es su centralidad. La Tabla 2 a su vez expone los términos que se analizaron, con el objetivo de corroborar su carácter central. La confirmación de la centralidad de un componente está dada por la cualidad que tiene dicho componente de ser un elemento fundamental de la RS, esto es, por la capacidad para otorgarle significado a toda la organización estructural. En palabras de Parales, "solamente los elementos que se consideren abso- lutamente esenciales para estructurar las RS pueden considerarse componentes del sistema central" (2006, p. 617).

Para verificar la centralidad de los elementos, se desarrolló el método del cuestionario de verificación (Guimelli, 2001) o cuestionamiento de centralidad (Parales, 2006). El cuestionario se desarrolló con un total de 42 estudiantes. En la Tabla 3 se encuentran las frecuencias con respecto a la cantidad de estudiantes que desarrollaron el cuestionario y los porcentajes de los elementos evaluados. 
Tabla 3. Posibles elementos centrales

\begin{tabular}{|l|c|c|c|}
\hline \multicolumn{1}{|c|}{ Palabra } & $\begin{array}{c}\text { Es esencial/importante } \\
\text { en la clase de ERE } \\
\text { (\% y frecuencia) }\end{array}$ & $\begin{array}{c}\text { No es esencial/ } \\
\text { importante en clase de } \\
\text { ERE } \\
\text { (\% y frecuencia) }\end{array}$ & $\begin{array}{c}\text { Tal vez } \\
\text { (\% y frecuencia) }\end{array}$ \\
\hline Religiones & $85,7 \%(36)$ & $9,5 \%(4)$ & $4,8 \%(2)$ \\
\hline Catolicismo & $76,2 \%(32)$ & $9,5 \%(4)$ & $14,3 \%(6)$ \\
\hline Fe & $76,2 \%(32)$ & $9,5 \%(4)$ & $14,3 \%(6)$ \\
\hline Dios & $73,8 \%(31)$ & $7,1 \%(3)$ & $19 \%(8)$ \\
\hline Moral, ética y valores & $73,8 \%(31)$ & $4,8 \%(2)$ & $21,4 \%(9)$ \\
\hline Conocimiento & $66,7 \%(28)$ & $9,5 \%(4)$ & $23,8 \%(10)$ \\
\hline Jesús & $66,7 \%(28)$ & $7,1 \%(3)$ & $26,2 \%(11)$ \\
\hline Enseñanza & $64,3 \%(27)$ & $7,1 \%(3)$ & $28,6 \%(12)$ \\
\hline Vida & $61,9 \%(26)$ & $14,2 \%(6)$ & $23,8 \%(10)$ \\
\hline Reflexión & $61,9 \%(26)$ & $7,1 \%(3)$ & $31 \%(13)$ \\
\hline Historia & $59.5 \%(25)$ & $16,6 \%(7)$ & $23,8 \%(10)$ \\
\hline Biblia & $54,8 \%(23)$ & $26,2 \%(11)$ & $19 \%(8)$ \\
\hline Creencias & $40,5 \%(17)$ & $26,1 \%(11)$ & $33,3 \%(14)$ \\
\hline
\end{tabular}

Fuente: elaboración propia con base en las respuestas de los estudiantes.

Al considerar los resultados del cuestionario de centralidad junto con los indicadores iniciales de la frecuencia, el rango y la importancia, se establecieron finalmente los elementos centrales y periféricos de las RS sobre la clase de ERE. Para alcanzar este objetivo se propusieron los siguientes criterios: un elemento puede considerarse central si la frecuencia es alta, si en el promedio de la población que usa la palabra es evocado en los primeros lugares, si el grado de importancia es relevante y si en el resultado del cuestionario el porcentaje de aprobación dado es alto. Según esto, se consideraron elementos periféricos aquellos con frecuencias altas que, sin embargo, en algunos de los indicadores restantes tuvieron valores bajos.
De modo específico, los criterios que se establecieron para determinar los valores que indican la relevancia de cada uno de los indicadores fueron los siguientes: la frecuencia se consideró relevante si la palabra fue mencionada cuatro veces o más por cada uno de los grupos y se consideró altamente significativa si más del 50\% de la población la utilizó. El rango se consideró alto si las palabras fueron evocadas dentro de los primeros ocho lugares. Este valor se estableció sobre el promedio de palabras usadas (si el promedio fue de 16 palabras, se consideraron relevantes aquellas mencionadas en los primeros lugares, en este caso, hasta el octavo lugar).

El grado de importancia se determinó solicitando a los estudiantes que escribieran en un es- 
quema predeterminado, dentro del taller asociativo, las cuatro palabras más relevantes para ellos. Finalmente, para determinar los valores del cuestionario de verificación se consideraron altamente relevantes los elementos que obtuvieron un porcentaje de aceptación por encima del 50\% y a este se adicionó el valor medio estipulado a la opción TAL VEz, pues se consideró viable establecer que este valor no descar- ta del todo la opción y en consecuencia puede corresponder, por lo menos en un porcentaje medio, a la opción sí.

Los resultados que se exponen en las Tablas 4 y 5 esquematizan el análisis de la relación de los indicadores y corresponden respectivamente a los elementos centrales de las RS sobre la clase de ERE (el núcleo central de la RS) y los elementos periféricos.

Tabla 4. El núcleo central de las RS sobre la clase de ERE (elementos verificados)

\begin{tabular}{|l|c|c|c|c|}
\hline \multicolumn{1}{|c|}{ Núcleo central } & Frecuencia & Rango & $\begin{array}{c}\text { Cuestionario de } \\
\text { verificación }\end{array}$ \\
\hline Dios & 34 & 4,27 & 29 & $73,8 \%$ \\
\hline Biblia & 33 & 5,52 & 16 & $54,8 \%$ \\
\hline Religiones & 23 & 6,19 & 7 & $85,7 \%$ \\
\hline Moral, ética y valores & 20 & 8,25 & 6 & $73,8 \%$ \\
\hline Jesús & 18 & 7,61 & 10 & $66,7 \%$ \\
\hline Vida & 18 & 6,89 & 4 & $61,9 \%$ \\
\hline
\end{tabular}

Fuente: elaboración propia con base en las respuestas de los estudiantes.

Tabla 5. Elementos periféricos de la RS sobre la clase de ERE

\begin{tabular}{|c|c|c|c|c|}
\hline Elementos periféricos & Frecuencia & Rango & Importancia & $\begin{array}{c}\text { Cuestionario de } \\
\text { verificación }\end{array}$ \\
\hline Fe & 20 & 6,90 & 11 & $76,2 \%$ \\
\hline Creencias & 18 & 6,94 & 14 & $40,5 \%$ \\
\hline Historia & 13 & 8,00 & 6 & $59,5 \%$ \\
\hline Reflexión & 10 & 7,80 & 3 & $61,9 \%$ \\
\hline Catolicismo & 10 & 6,80 & 0 & $76,2 \%$ \\
\hline Enseñanza & 9 & 4,78 & 8 & $64,3 \%$ \\
\hline Conocimiento & 8 & 5,50 & 3 & $66,7 \%$ \\
\hline
\end{tabular}

Fuente: elaboración propia con base en las respuestas de los estudiantes. 
Posteriormente, siguiendo la propuesta de $\mathrm{Mi}$ reles (2015) de agrupar conceptos en grandes categorías para organizar la estructura de las RS, al examinar y organizar las palabras del núcleo central y las periféricas se descubrió que podían organizarse en cuatro categorías más amplias: lo confesional, lo plural, lo ético-moral y lo académico. En torno a estas se agruparon las palabras restantes, obtenidas en el ejercicio asociativo, pero que aparecieron con menor frecuencia y peso semántico. Esta acción se desarrolló con la intención de mostrar un conjunto más amplio de palabras que los estudiantes evocaron a propósito de la clase de ERE y conocer otros términos que se articulaban a los elementos ya constituidos de las RS.

\section{Aproximación al enfoque procesual de las RS sobre la ERE (análisis cualitativo)}

La recolección de los datos de orden cualitativo se desarrolló a través de los grupos de discusión, en los cuales se propusieron cinco preguntas que se derivaron de las palabras o elementos centrales que resultaron del taller asociativo. Al hacer la revisión de las respuestas, se encontraron los siguientes aspectos: sobre la primera pregunta: ¿qué es la clase de ERE?, los temas sobresalientes fueron: el conocimiento y respeto hacia otras creencias, la formación ética y moral, el respeto a otras religiones, la orientación de la dimensión espiritual, la formación en principios y valores y la relación con Dios. Frente a la segunda pregunta: ¿qué se enseña en la clase de ERE y qué debería enseñarse?, en el conjunto de los temas más significativos nombrados por los estudiantes se encontraron: la planeación del proyecto de vida, la búsqueda de sentido e identidad, el reconocimiento de otras religiones y la enseñanza de cuestiones filosóficas.

Con respecto a la tercera pregunta de discusión: ¿cómo es una clase de ERE y cómo debería ser?, frente a la primera parte de la pregunta, las respuestas se enfocaron en el aspecto metodológico de la enseñanza de la ERE y se usaron palabras como reflexiva, amena, divertida, teórica, con debates e histórica. Sobre la segunda parte de la pregunta, las contestaciones se focalizaron más en el contenido que en el componente metodológico de la ERE y se hizo referencia a palabras y expresiones como el componente social de la ERE, la autoestima y la autosuperación para alcanzar sentido, la felicidad personal, el estudio de otras religiones y la libertad religiosa.

En relación con la pregunta número cuatro: ¿en la clase de ERE se enseña, se promueve y se respeta el pluralismo religioso?, se mencionaron palabras y frases como el conocimiento de otras religiones, el predominio de la religión católica y el respeto a la expresión de las diversas creencias y prácticas religiosas. Finalmente, sobre la quinta pregunta, acerca de la importancia de la ERE, el elemento predominante residió en que los participantes hicieron eco de las grandes categorías abordadas en los apartados anteriores. Los términos o expresiones usados fueron: conocimiento de Dios, de otras religiones y de sí mismo, guía para tomar decisiones, bases para el proyecto de vida y educación ética y moral.

A partir del análisis de los datos expuestos en esta sección se identificaron algunos de los elementos constitutivos de las RS sobre la clase de ERE. En primer lugar, se reconoció que, al igual que los resultados obtenidos del análisis estructural, las categorías de lo confesional, lo plural, lo ético-moral y lo académico también se presentaron en el análisis de los datos obtenidos en los grupos de discusión, aunque la última categoría apareció con menor fuerza. En segundo lugar, el elemento que se manifestó con mayor fuerza en la estructura de las RS sobre la ERE es el pluralismo religioso y su relación directa con la necesidad de reconocimiento de otras religiones distintas al catolicismo. En tercer lugar, emergió un elemento que hasta ahora no había contado como relevante y es la categoría de proyecto de vida, en el que se vincularon conceptos como sentido de la vida, felicidad e identidad. 


\section{Diálogo entre los enfoques estructural y procesual}

Retomando el camino metodológico propuesto para la investigación, que pone en diálogo los aportes de los enfoques procesual y estructural, el siguiente paso en el trabajo consistió en cruzar la información obtenida a través de las técnicas narrativas y asociativas, articulando el análisis a los componentes esenciales que, desde la perspectiva de Moscovici (1979), constituyen las RS y que en la propuesta de Gómez (2000) fueron asumidas como unidades de análisis: las dimensiones de información, campo de representación y actitud. Con esta guía se revisaron y analizaron los fragmentos de los grupos de discusión.

DIMENSIÓN DE INFORMACIÓN: grosso modo, la dimensión de información corresponde a "la organización de los conocimientos que posee un grupo con respecto a un objeto social, es decir, son los conocimientos que tienen los sujetos" (Flores, 2010, p. 85). En el caso de la técnica narrativa, la dimensión de información se organizó esencialmente en torno a las respuestas que dieron los estudiantes a las preguntas qué es y qué se enseña en la clase de ERE, respuestas que se lograron agrupar mayoritariamente en torno a los conceptos de pluralismo religioso, formación ética, moraly en valores, proyecto de vida y catolicismo.

En general, al poner en diálogo las técnicas de corte asociativo y narrativo, se encontró que la coherencia entre la información obtenida por medio de estas técnicas se conservó, de tal manera que la información expresada por los estudiantes siempre relacionó los conceptos de pluralismo religioso, formación ética, moral y en valores, proyecto de vida y catolicismo como elementos característicos de la clase de ERE. Aunque la información se relacionó y fue coherente, varió la intensidad y frecuencia de las relaciones entre las palabras y frases expresadas en la técnica narrativa con los componentes estructurales del núcleo central y los elementos periféricos.
Por ejemplo, en la información manifestada por los estudiantes efectivamente se relacionó el pluralismo religioso con la clase de ERE, en primer lugar, con mayor intensidad y frecuencia, con el término religiones (elemento constitutivo del núcleo central identificado en el análisis estructural); en segundo lugar, con el término creencias (componente de los elementos periféricos); finalmente y con menor fuerza, se nombraron otras expresiones y palabras como hinduismo, judaismo, cristianismo, espiritualidad, culturas, tradiciones, pluralismo, diferentes ritos e identidades religiosas, que hacen parte del grupo de palabras más extenso que los estudiantes evocaron sobre la clase de ERE.

Con estos datos fue posible caracterizar el conjunto de conocimientos -dimensión de informaciónque poseen los sujetos sobre la ERE. En los resultados se muestran fuertes y constantes referencias a los conceptos de pluralismo religioso, proyecto de vida y formación ética, moral y en valores. Con menor intensidad y frecuencia se estableció la relación con la palabra catolicismo. Aunque el concepto proyecto de vida no emergió como central en el análisis estructural -técnicas asociativas-, a partir del análisis hecho con base en la técnica narrativa se manifestó con gran relevancia, frecuencia e intensidad. Finalmente, la referencia al catolicismo se presentó como un cuestionamiento a su predominio en la clase de ERE, la mayoría de las referencias al concepto discutieron la escasa inclusión de otras experiencias religiosas en la clase.

DIMENSIÓN CAMPO DE REPRESENTACIÓN: en esta dimensión se encuentran los componentes que estructuran las RS; en concreto se refiere a la ordenación y jerarquización de las partes que configuran su contenido. Además, esta dimensión se establece alrededor de un "esquema o núcleo figurativo que es construido en los procesos de objetivación y anclaje" (Jodelet, 1986, citado en Flores, 2010, p. 82). Para estructurar esta dimensión se tomaron nuevamente los grupos de palabras que en el análisis cuantitativo ayudaron a configurar, ordenar y jerarquizar los 
elementos de la RS: lo confesional, lo plural, lo éticomoral y lo académico. A través de estos conceptos las RS sobre la clase de ERE se hicieron concretas y comprensibles a los sujetos. Sin embargo, se encontró que, en dicha ordenación y jerarquización de los componentes de la RS, tanto en el ejercicio asociativo como en el discurso de los estudiantes a través de los grupos de discusión, se consolidó el predominio de tres grupos dentro de la representación y se manifestó un concepto nuevo.

Con base en el análisis cuantitativo se constituyeron los elementos del núcleo central de las RS sobre la ERE en las siguientes palabras: Dios/dios, biblia, religiones, moral-ética-valores, Jesús y vida. De igual manera se establecieron los elementos periféricos: fe, creencias, historia, reflexión, catolicismo, enseñanza y conocimiento. Continuando con la propuesta de análisis, estos términos se acoplaron a los grandes grupos de palabras de la siguiente manera: en el núcleo central prevalece lo plural (dios y religiones), lo confesional (Dios, biblia, Jesús) y lo éticomoral (moral, ética y valores). En los elementos periféricos, predomina lo académico (historia, reflexión, enseñanza y conocimiento) y también hay una presencia menor de lo plural (fe y creencias) y de lo confesional (catolicismo).

Contrapuesta esta información con los datos del análisis cualitativo desarrollado sobre los grupos de discusión, se encontró que los elementos que prevalecen y en los que coinciden las narrativas de los estudiantes fueron lo plural, lo ético-moral y el proyecto de vida. Aunque este último elemento emergió de manera especial en esta instancia de la reflexión, en el análisis cuantitativo y específicamente en las palabras del núcleo central se estaba haciendo latente con la palabra vida. En una segunda instancia y con menos fuerza aparecen los elementos en relación con lo académico y nuevamente con lo confesional.

A modo de cierre, se encontró, en relación con el campo de representación, que sobre este se or- denan y jerarquizan los elementos de las RS sobre la ERE de la siguiente manera: en el grupo de los elementos centrales se localizaron lo plural, lo ético-moral, el proyecto de vida y lo confesional. En el conjunto de los elementos periféricos se ubicaron lo académico y, en parte, lo confesional y lo plural. Cada uno de estos grandes grupos se manifestó a través de los términos centrales y periféricos identificados por medio del análisis estructural, y en el caso específico del proyecto de vida, se dio mediante palabras y expresiones reconocidas en el análisis cualitativo de los grupos de discusión.

DIMENSIÓN DE ACTITUDES: esta dimensión se refiere a la orientación general respecto al objeto de las RS, orientación que puede ser favorable o desfavorable, positiva o negativa. No obstante, la actitud no es una simple reacción, sino que es una producción de comportamientos. La actitud, como componente de las RS, mueve a determinados comportamientos con respecto a su objeto. Según esto, para identificar esta dimensión en las RS de los estudiantes sobre la clase de ERE, se analizaron los términos y/o expresiones favorables y desfavorables, positivos y negativos hacia la clase, expresados/as tanto en el ejercicio asociativo como en los grupos de discusión.

Con base en el taller asociativo, se tuvieron en cuenta las palabras más recurrentes, que fueron las siguientes: obligación, imposición, aburrido y homogéneo. A partir del análisis acerca de los grupos de discusión se desglosaron los siguientes términos: imposición, importante, adecuada, respeta la diversidad, amena, divertida y relajada. También, en el cuestionario de verificación se adicionaron dos preguntas que reflejaban actitudes sobre la clase, con énfasis en la importancia y grado de interés que genera la clase, hasta obtener porcentajes de aprobación respectivamente de $61,9 \%$ y $88,1 \%$.

De acuerdo con lo anterior, se organizaron los conceptos de la siguiente manera: dentro de las actitudes favorables sobre la clase de ERE se ubica- 
ron: importante, adecuada, respeto por la diversidad, agradable, amena y divertida. En general, estas palabras hacen referencia a los contenidos y a los elementos metodológicos de la clase. En el conjunto de las actitudes desfavorables se ubicaron los términos obligación, imposición, aburrido y relajada. Los términos obligación e imposición se mencionaron en relación con los contenidos de la clase, determinados y establecidos con cierto exclusivismo desde la religión católica. Las palabras aburrido y relajado fueron referidas tanto a los contenidos como a los métodos de la clase, en ocasiones repetitivos, monótonos y poco innovadores.

Según W. Doise (1986), la dimensión de las actitudes es sustancial en la construcción de las RS, porque "permite asumir una postura frente a algo" (citado por Flores, 2010, p. 89). En cuanto a la RS sobre la clase de ERE, ningún conjunto de actitudes predomina sobre el otro y ambos tienen el mismo peso en relación con las RS de los estudiantes sobre la clase. En general, las actitudes develadas son reflejo de las diversas experiencias de los estudiantes frente a la ERE, que a su vez determinan los comportamientos y prácticas en la clase. Estas se caracterizan por un movimiento flexible entre el interés y el desinterés, entre la motivación y el desánimo, entre el aprecio y la subestimación, consecuencia de la metodología y los contenidos propuestos desde la clase de ERE.

\section{Una clase católica pero pluralista: sintesis de las RS sobre la clase de ERE}

Considerando en conjunto los elementos brindados desde la perspectiva estructural y procesual, se presentan ahora las RS sobre la clase de ERE. La primera característica que sobresale en la estructura de las RS sobre la ERE se deriva del análisis y la clasificación de las palabras del núcleo central y las palabras periféricas, pues se encontró que estas podían agruparse en cuatro grandes categorías: lo plural, lo éticomoral, lo confesional y lo académico. De este modo se puede afirmar que los estudiantes, al aprehender y construir la clase de ERE como objeto representado, lo hacen mediante estos conceptos. Ahora bien, discriminando los términos centrales de los elementos periféricos, se halló que en el núcleo de las RS se encuentran las categorías de lo plural, lo ético-moral y lo confesional, mientras que en los elementos periféricos se ubica la categoría de lo académico.

Como consecuencia de este análisis dialogal entre los enfoques estructural y procesual, posteriormente se develó otra categoría como parte del núcleo central de las RS sobre la clase de ERE: proyecto de vida. Respecto a esta categoría, en los instrumentos asociativos se hizo mención constante de la palabra vida y en los grupos de discusión específicamente de la expresión proyecto de vida. Al revisar las respuestas dadas en los grupos de discusión y tras el análisis de las narrativas, se verificaron los elementos centrales y periféricos antes mencionados; sin embargo, a partir de este análisis emergió la nueva categoría y característica de las RS sobre la ERE que tiene que ver con proyecto de vida y que a su vez se relacionó directamente con los conceptos de felicidad y sentido.

A este respecto se puede afirmar que el conocimiento social que tienen los estudiantes sobre la clase de ERE a través de las RS se ordena y jerarquiza de la siguiente manera: el primer elemento que estructura las RS es el componente confesional de la ERE, pues ciertamente es una clase dada en instituciones católicas, situación que determina el enfoque de enseñanza; el segundo elemento es el componente plural, según el cual, aunque se enseñe religión católica como la religión de la institución, también se enseñan otras religiones y se espera que adquieran la misma importancia que la religión institucional; en tercer y cuarto lugar, respectivamente, están el elemento ético-moral y el proyecto de vida, dado que en la ERE se proporcionan herramientas conceptuales y prácticas fundamentales para la construcción de valores y para la estructuración del proyecto de vida. Finalmente, se piensa en lo académico, dado que la ERE, como disciplina escolar, desarrolla procesos pedagógicos para la enseñanza y aprendizaje del saber que le es propio. 
Por otra parte, y teniendo en cuenta que la construcción de un sentido global sintetiza y especifica la forma en que los sujetos apropian y construyen el objeto representado, lo que es para ellos y cómo se sitúan frente a esa construcción (Singéry, 2001), la segunda característica de las RS sobre la clase de ERE, en estrecha relación con lo mencionado en la primera, se puede expresar de la siguiente manera: la clase de ERE está representada en primer lugar por uno de los temas de conocimiento de esta área del saber: Dios, término acompañado muy de cerca por la palabra Biblia, entendida, en la visión cristiana católica, como el medio o instrumento para acceder al conocimiento del misterio de lo divino, a la vez relacionado con la palabra Jesús, con la que se hace un énfasis en el carácter cristiano católico de la ERE (enfoque confesional en la RS sobre la clase de ERE).

La referencia a las demás religiones corresponde al reconocimiento de la existencia de otras experiencias espirituales y religiosas que también deben ser estudiadas en una clase que sea propiamente de educación religiosa. Aunque parece coherente que en un colegio católico se enseñe la religión católica, también se espera que en la clase de educación religiosa se promueva el estudio de otras religiones en su riqueza histórica, cultural, espiritual, etc. (componente plural de las RS sobre la ERE). La moral, la ética $y$ los valores son los criterios o parámetros que ayudan a configurar el estilo de vida y comportamiento de los seres humanos, por lo que correspondería a la ERE incidir de manera concreta y práctica en la cimentación del proyecto de vida de los estudiantes que se forman en la clase (referencia a los elementos ético-moral y proyecto de vida en las RS sobre la ERE).

Finalmente, la mención, por una parte, de las palabras fe, historia y creencias y, por otra, de los términos reflexión, enseñanza y conocimiento, se resaltan como características conceptuales y metodológicas de la clase de ERE, que, en cuanto disciplina escolar, se ocupa del estudio de la realidad religiosa y de la estructuración del conocimiento sobre la ex- periencia religiosa; y como área de formación, aporta con la educación integral de los seres humanos, con la búsqueda de sentido y con la formación en valores (componente académico en las RS sobre la clase de ERE).

\section{Conclusiones}

Al finalizar este proceso investigativo, se puede concluir que las características identificadas en las RS sobre la clase de ERE no corresponden a una visión exclusivamente confesional y tradicional de la clase. Al referirse a la ERE en un contexto escolar católico, con bastante probabilidad se puede pensar que los elementos que la organizan son eminentemente de corte confesional; sin embargo, las RS develadas a partir de las voces de los estudiantes permitieron descubrir que, aunque hay una fuerte presencia de la enseñanza del catolicismo, elemento que en el análisis cualitativo y cuantitativo es central en las RS, también se posibilita el conocimiento de otros elementos dentro de la ERE que no son únicamente católicos y que abordan aspectos que incluyen a otras religiones, experiencias espirituales y trascendentales, tales como la oración, la libertad, la felicidad, la paz, la sabiduría y la verdad.

Por otra parte, las RS sobre la clase de ERE manifiestan una estrecha relación con la enseñanza de la religión católica en el contexto escolar, pero al mismo tiempo develan una tensión entre el estudiante, quien tiene unos intereses y deseos, y la propuesta académica institucional sobre la clase de ERE. Esta tensión se expresa entre lo plural exteriorizado por los estudiantes y lo confesional propuesto por las instituciones educativas católicas. Esta situación se resuelve, por una parte, asumiendo que la ERE que se enseña en una institución católica debe desarrollar elementos propios de la religión institucional y, por otra, justificando y reclamando que una clase de ERE en cuanto tal, al ocuparse del hecho religioso, debe involucrar también componentes de las demás tradiciones espirituales y religiosas. 
En este sentido, se afirma que la principal característica de las RS sobre la clase de ERE, no la única, es que la ERE es una clase confesional, pero pluralista. En los datos analizados desde los enfoques procesual y estructural, los estudiantes manifestaron constantemente que en la clase se desarrollan elementos propios de una educación religiosa católica, aunque también, y con menor intensidad, se trabajan elementos de otras religiones. En este punto se comprendió que la ERE se desarrolla con cierta tensión entre lo que es y lo que debería ser: la clase de ERE es una clase de religión católica donde se desarrollan algunos temas y actividades en relación con las demás religiones, pero ella debería desarrollar en igualdad de condiciones lo relacionado con todas las religiones y experiencias espirituales, es decir, hacer posible una relación de equidad en el ámbito de la clase.
Finalmente, frente a lo develado en las RS sobre ERE, resultan significativos los siguientes elementos: primero, de manera explícita se establece una separación entre ERE y religión católica, de tal manera que hablar de ERE no significa necesariamente hablar de religión católica. Segundo, el reconocimiento del valor de las otras religiones, que incluya la riqueza histórica, cultural y espiritual de cada una de ellas en la enseñanza de una ERE propiamente dicha. Por último, se afirma la importancia de justificar la ERE sobre una base distinta a la propuesta exclusivamente centrada en una religión, un cimiento más humano y universal para que, indistintamente de la institución religiosa, el hecho religioso contribuya con la educación integral y la formación espiritual de las personas.

\section{Referencias}

Abric, J. C. (2001). Prácticas sociales y representaciones. México: Coyoacán.

Alasino, N. (2011). Alcances del concepto de representaciones sociales para la investigación en el campo de la educación. Revista Iberoamericana de Educación, 56(4), 1-11. DOI: https://doi.org/10.35362/rie5641500

Araya, S. (2002). Las representaciones sociales: ejes teóricos para su discusión. San José, Costa Rica: Flacso.

Asamblea Nacional Constituyente (1991). Constitución Política de Colombia. http://www.constitucioncolombia. com/indice.php

Banchs, M. A. (1988). Cognición social y representación social. Psicología de El Salvador, 7(30), 361-371.

Benjumea-Loaiza, C. y Vélez-Vélez, J. (2021). Representaciones sociales de Educación Religiosa en la media académica: una apuesta desde la virtualidad. Educación y Humanismo, 23(41), 119-137. DOI: https://doi. org/10.17081/eduhum.23.41.4921

Bonilla, J. (ed.) (2013). Reflexiones y perspectivas sobre educación religiosa escolar. Bogotá: Bonaventuriana.

Bonilla, J. (ed.) (2014). Educación religiosa escolar, pedagogías para el reconocimiento del pluralismo religioso. Bogotá: Bonaventuriana.

Bonilla, J. (2015). Educación religiosa escolar en perspectiva de complejidad. Bogotá: Bonaventuriana. 
Botero, C. y Hernández, A. (ed.) (2018). Approaches to the nature and epistemological foundations: Of religious education in Colombian schools. Bogotá: USTA. DOI: https://doi.org/10.2307/j.ctvr33dht

Cantillo, D. y Quintero, F. (2020). Aportes de la educación religiosa escolar a la promoción del pluralismo religioso. Hojas y Hablas, 20, 84-96. DOI: https://doi.org/10.29151/hojasyhablas.n20a6

Conferencia Episcopal de Colombia (2017). Estándares para la Educación Religiosa Escolar. Bogotá: Delfín.

Congreso de la República de Colombia (1994). Ley 115 del 8 de febrero. http://www.mineducacion.gov.co/1621/ articles-85906_archivo_pdf.pdf

Coy, M. (2009). Educación religiosa escolar: ¿por qué y para qué? Franciscanum, 51(152), 49-70. https://revistas. usb.edu.co/index.php/Franciscanum/article/view/953

Coy, M. (2010). La educación religiosa escolar en un contexto plural. Franciscanum, 52(154), 53-83. http://revistas. usbbog.edu.co/index.php/Franciscanum/article/view/943

Cuellar, N. y Moncada, C. (ed.) (2019). La educación religiosa como disciplina escolar en Colombia. Cali: Unicatólica. https://editorial.unicatolica.edu.co/omp/index.php/Sello_Editorial/catalog/book/53

Cuellar, N., Moncada, C. y Valencia, W. (comps.) (2020). Currículo en ERE: orientaciones para su reflexión, fundamentación, diseño e innovación. Cali: Unicatólica. https://editorial.unicatolica.edu.co/omp/index.php/ Sello_Editorial/catalog/book/84

Cuevas, Y. (2016). Recomendaciones para el estudio de representaciones sociales en investigación educativa. Cultura y Representacionnes Sociales, 11(21), 109-140. http://www.scielo.org.mx/scielo.php?script=sci_artte $x t \& p i d=\$ 2007-81102016000200109$

Flores, R. (2010). Educación ambiental, representaciones sociales del uso del agua. Revista Educación y Desarrollo Social, 4(1), 78-93. https://revistas.unimilitar.edu.co/index.php/reds/article/view/916/670

Gómez-Mendoza, M. Á. (2000). Análisis de contenido cualitativo y cuantitativo. Definición, clasificación y metodología. Revista de Ciencias Humanas, 20, 129-138. http://www.utp.edu.co/ chumanas/revistas/revistas/ rev2o/gomez.htm

Guimelli, C. (2001). La función de enfermera. Prácticas y representaciones sociales. En J. C. Abric (coord.), Prácticas sociales y representaciones (pp. 75-96). México: Coyoacán.

Jodelet, D. (1984). La representación social: fenómenos, conceptos y teoría. En S. Moscovici, Psicología social II. Pensamiento y vida social. Psicología social y problemas (pp. 469-494). Barcelona-Buenos Aires-México: Paidós.

Jodelet, D. (2011). Aportes del enfoque de las representaciones sociales al campo de la educación. Espacios en Blanco. Revista de Educación, 21, 133-154. https://www.redalyc.org/pdf/3845/384539803006.pdf

Meza, J. et al. (2011). Educación religiosa escolar. Naturaleza, fundamentos y perspectivas. Bogotá: Pontificia Universidad Javeriana. 
Meza, J. et al. (2013). Educación religiosa escolar en clave liberadora: elementos constitutivos. Revista Theologica Xaveriana, 63/1(175), 219-248. https://revistas.javeriana.edu.co/index.php/teoxaveriana/article/view/9322

Mireles, O. (2015). Metodología de la investigación: operaciones para develar representaciones sociales. Magis, Revista Internacional de Investigación en Educación, 8(16), 149-166. DOI: https://doi.org/10.11144/Javeriana. m8-16.miop

Moncada, C. (2020). Perspectivas de la educación religiosa escolar desde los estudios de la religión. Bogotá: USTA. DOI: https://doi.org/10.15332/li.lib.2020.00216

Moncada, C. y Pérez, J. (2020). Análisis de la educación religiosa escolar desde la normatividad colombiana como aporte a la reflexión educativa en Latinoamérica. En J. Pérez, C. Pinto, C. Moncada, J. Nieto y J. SantamaríaRodríguez, Reflexiones alrededor de la Iglesia y la educación en el contexto latinoamericano (pp. 6o-81). Córdoba: Universidad Católica de Córdoba. http://pa.bibdigital.uccor.edu.ar/2181/

Moscovici, S. (1979). El psicoanálisis, su imagen y su público. Buenos Aires: Huemul.

Naranjo, S. y Moncada, C. (2019). Aportes de la educación religiosa escolar al cultivo de la espiritualidad humana. Revista Educación y Educadores, 22(1), 103-119. DOI: https://doi.org/10.5294/edu.2019.22.1.6

Ortiz, J. y Quintero, F. (2020). Mooc y pedagogía: Encuentros y desencuentros desde la investigación. En. J. Rojas y J. Ortíz (eds.), Reflexiones metodológicas de investigación educativa: perspectivas tecnológicas (pp. 108-149). Bogotá: USTA. https://repository.usta.edu.co/bitstream/handle/11634/31293/Obracompleta. Coleccion440.2020Rojasjulio.pdf?sequence $=4 \&$ isAllowed $=y$

Parales, C. (2006). Representaciones sociales sobre el comer saludable: un estudio empírico en Colombia. Universitas Psychologica, 5(3), 613-626. http://pepsic.bvsalud.org/pdf/up/v5n3/v5n3a15.pdf

Pérez, J., Nieto-Bravo, J. y Santamaría-Rodríguez, J. (2019). La hermenéutica y la fenomenología en la investigación en ciencias humanas y sociales. Revista Civilizar: Ciencias Sociales y Humanas, 19(37). 21-29. DOI: https://doi.org/10.22518/usergioa/jour/ccsh/2019.2/aog

Pérez, J., Pinto, C., Moncada, C., Nieto, J. y Santamaría-Rodríguez, J. (2020). Reflexiones alrededor de la Iglesia y la educación en el contexto latinoamericano. Córdoba: Universidad Católica de Córdoba. http://pa.bibdigital. uccor.edu.ar/2181/

Pérez, J., Ramírez, J. y Rojas, J. (2020). La investigación documental como sustento de la investigación social y educativa. En J. Pérez y J. Nieto, Reflexiones metodológicas de investigación educativa: Perspectivas sociales (pp. 105-135). Bogotá: USTA. https://repository.usta.edu.co/bitstream/handle/11634/31292/Obracompleta. Coleccion440.2020Perezjohn.pdf?sequence $=4 \&$ isAllowed $=y$

Quintero, F.y Ortiz, J. G. (2020). Representaciones sociales: una perspectiva metodológica para la investigación educativa. En J. Pérez y J. Nieto (coords.), Reflexiones metodológicas de investigación educativa. Perspectivas sociales (57-104). Bogotá: USTA. https://repository.usta.edu.co/bitstream/handle/11634/31292/Obracompleta.Coleccion440.2020Perezjohn.pdf?sequence $=4$ \&isAllowed $=y$ 
ISSN 0123-1294 | e-ISSN 2027-5358 | Educ.Educ. Vol. 24. No.3 | Agosto-octubre de 2021 | e2433

Universidad de La Sabana | Facultad de Educación

Rateau, P. y Monaco, G. L. (2013). La teoría de las representaciones sociales: orientaciones conceptuales, campos de aplicaciones y métodos. Revista CES Psicología, 6(1), 22-42. http://www.scielo.org.co/pdf/cesp/v6n1/ v6n1ao3.pdf

Rodríguez, C., Lorenzo, O. y Herrera, L. (2005). Teoría y práctica del análisis de datos cualitativos. Proceso general y criterios de calidad. Revista Internacional de Ciencias Sociales y Humanidades: Sociotam, 15(2), 133-154. https://www.redalyc.org/pdf/654/65415209.pdf

Singéry, J. (2001). Representaciones sociales y proyecto de cambio tecnológico en empresa. En J. C. Abric (coord.), Prácticas sociales y representaciones (pp. 159-194). México: Coyoacán. 